REVESCO. Revista de Estudios Cooperativos ISSN: $1885-8031$

http://dx.doi.org/10.5209/REVE.61937

\title{
La Educación como vía de empoderamiento laboral y social de la juventud universitaria en México
}

\author{
Celia Fausto Lizaola ${ }^{1}$, Juan Carlos Tójar Hurtado² y José Manuel Ríos Ariza ${ }^{3}$
}

Recibido: 2 de julio de 2018 / Aceptado: 4 de octubre de 2018

Resumen. La educación es el eje estratégico para activar la transformación económica y cultural de las naciones, promover la calidad de vida de la sociedad y la vinculación de las personas con el mundo del trabajo. Invertir individual y socialmente en educación, genera beneficios de rentabilidad macrosocial, microsocial y personal. De este modo, el futuro personal y colectivo, está ligado al proceso educativo y a la formación de personas críticas, que puedan intervenir en la mejora de su realidad social (Díaz Domínguez y Alemán, 2008). La educación, y en especial la superior, prepara a la ciudadanía para la vida, generando altas expectativas individuales y colectivas, para construir un mejor entorno social (Soto y Torres, 2016). Con un nivel educativo óptimo, la juventud contribuye a la economía nacional y a reducir la brecha de desigualdad social (OCDE/CEPAL/ CAF, 2016). Desde esta perspectiva, las Instituciones de Educación Superior (IES), tienen el compromiso con la sociedad de reforzar el binomio calidad-pertinencia. Todo ello para que la juventud mexicana cuente con el perfil profesional que exigen los retos y expectativas de la realidad social. Frente a este panorama, el planteamiento es: ¿La formación universitaria contribuye al empoderamiento laboral y social de la juventud mexicana? Para este estudio se han considerado cuatro centros de la Universidad de Guadalajara, de diferentes regiones del Estado de Jalisco, México. Con este trabajo se busca analizar la fiabilidad y validez de la evaluación realizada, con un instrumento orientado a analizar el entorno laboral y social del alumnado que egresa de la Universidad de Guadalajara. Se han elaborado análisis de consistencia interna (alpha de Cronbach) y factoriales (validez estructural). Los resultados destacan los factores más relevantes en relación al desarrollo de competencias durante su trayectoria formativa y, al mismo tiempo, avalan el uso del instrumento para evaluar líneas estratégicas orientadas a fortalecer los currícula académicos, de los centros universitarios objeto de estudio. Las conclusiones muestran cómo la formación universitaria recibida faculta a la población egresada para el empoderamiento social y, en menor medida, para el laboral y económico.

Palabras clave: Educación superior; Juventud; Población egresada; Competencias; Competencias emprendedoras.

Claves Econlit: I20; I23; A13; L26.

1 Universidad de Guadalajara, México

Dirección de correo electrónico: celiafausto@yahoo.com.mx

2 Universidad de Málaga, España

Dirección de correo electrónico: jctojar@uma.es

3 Universidad de Guadalajara, México

Dirección de correo electrónico: jmrios@valles.udg.mx 


\title{
[en] Education as a way of labor and social empowerment of university students in Mexico
}

\begin{abstract}
Education is the strategic axis to activate the economic and cultural transformation of nations, promote the quality of life of society and the connection of people with the world of work. Investing individually and socially in education, generates benefits of macro-social, microsocial and personal profitability. In this way, the personal and collective future is linked to the educational process and to the formation of critical people who can intervene in the improvement of their social reality (Díaz Domínguez \& Alemán, 2008). Education, and especially higher education, prepares citizens for life, generating high individual and collective expectations, to build a better social environment (Soto \& Torres, 2016). With an optimal level of education, young people contribute to the national economy and reduce the social inequality gap (OECD/CEPAL/CAF, 2016). From this perspective, the Institutions of Higher Education are committed to society to strengthen the binomial quality-relevance. All of the above is for Mexican youth to have the professional profile demanded by the challenges and expectations of the social reality. Against this background, the approach is: Does university education contribute to the social and labor empowerment of Mexican youth? For this study four educational centers of the University of Guadalajara, from different regions of the State of Jalisco, Mexico, have been considered. This work seeks to analyze the reliability and validity of the evaluation carried out, with an instrument aimed at finding out about the work and social environment of the students who graduate from the University of Guadalajara. Analyzes of internal consistency (Cronbach's alpha) and factorial (structural validity) have been developed. The results highlight the most relevant factors in relation to the development of competences during their formative trajectory and, at the same time, endorse the use of the instrument to evaluate strategic lines aimed at strengthening the academic curricula of the university centers under study. The conclusions show how the university education received trains graduates for social empowerment and, to a lesser extent, for labor and economic empowerment.
\end{abstract}

Keywords: Higher education; Youth; Graduates; Skills; Entrepreneurial competencies.

Sumario. 1. Introducción. 2. ¿La educación como solución y empoderamiento? La educación eje estratégico en discusión. 3. Metodología. 4. Resultados. 5. Conclusiones. 6. Referencias bibliográficas.

Cómo citar: Fausto Lizaola, C., Tójar Hurtado, J.C. y Ríos Ariza, J.M. (2018) La Educación como vía de empoderamiento laboral y social de la juventud universitaria en México. REVESCO. Revista de Estudios Cooperativos, Tercer Cuatrimestre, No 129, pp. 84-101. DOI: 10.5209/REVE.61937.

\section{Introducción}

Esta investigación se realiza en la Universidad de Guadalajara (UdeG), del Estado de Jalisco en México. Esta universidad pública es una de la más antiguas de América (se crea en 1792), y cuenta con una de las mayores poblaciones de estudiantes (280.297 según datos de la UdeG, 2018), e integra a estudiantes preuniversitarios y universitarios (123.932 estudiantes).

La docencia universitaria en la Universidad de Guadalajara es impartida por una red de Centros Universitarios, de ellos 6 están en el área metropolitana de Guadalajara y son temáticos (están aglutinados por áreas de conocimientos afines), y 9 situados en distintas regiones del Estado de Jalisco; éstos incluyen titulaciones de distintas áreas de conocimiento. Además, cuenta con un Sistema de Educación Virtual que imparte docencia online.

La Universidad de Guadalajara, como es usual en las universidades públicas, realiza de forma periódica una evaluación o reflexión sobre su labor, valorando los 
distintos servicios que presta en materia de docencia, investigación y extensión. Los informes oficiales realizados tienen un gran valor y aportan una estadística que permite responder a los indicadores que les exige la sociedad, a través de organizaciones mexicanas, como la Secretaría de Educación Pública (SEP), la Asociación Nacional de Universidades e Instituciones de Educación Superior (ANUIES) o el Consejo Nacional de Ciencia y Tecnología (CONACYT). La autoevaluación y los informes internos que realiza la Universidad de Guadalajara son importantes y relevantes, pero no invalidan la necesidad de buscar otras miradas, valoraciones e interpretaciones de la labor que no sean institucionales.

Esta investigación se va a centrar en la población egresada de la Universidad de Guadalajara en los últimos 5 años. La población egresada objeto del estudio pertenece a dos Centros Universitarios Temáticos y a dos Centros Universitarios Regionales, y entre estas personas solo se han seleccionado a quienes han cursado 8 licenciaturas. El criterio de selección fue incluir las titulaciones que se imparten en al menos dos de los Centros Universitarios, con objeto de poder establecer ciertas comparaciones.

La investigación se ha centrado en conocer en qué medida la formación recibida en los Centros Universitarios ha tenido efectos para su integración laboral y social. Además de la importancia local de este estudio, el mismo es relevante porque se incardina dentro de uno de los debates más relevantes (tanto social como científico), en la actualidad. Puesto que, por un lado, se insiste en la necesidad de la educación como una herramienta para garantizar el desarrollo de la humanidad y el desarrollo personal de los habitantes del planeta. Por otro lado, hay una crisis, fruto del no cumplimiento de las enormes expectativas que se habían depositado en la misma. Este debate, importancia, contradicciones y problema, colocan a la educación en un lugar central en la vida de la sociedad, como solución y como problema. Conocer, por tanto, la valoración de la población egresada de nuestras universidades cobra, en este contexto, especial relevancia.

Entre las limitaciones de la estadística pública de la Universidad de Guadalajara (2018) no se han encontrado datos de procedencia social de los estudiantes que ingresan y egresan en los estudios universitarios.

\section{2. ¿La educación como solución y empoderamiento? La educación eje estratégico en discusión}

La UNESCO (2009) considera la educación superior como un bien público. Asimismo, responsabiliza a la educación superior de avanzar en la comprensión de los complejos desafíos mundiales (presentes y futuros), sociales, científicos, económicos y culturales.

La labor social de la Universidad y de socialización está ampliamente aceptada, y debe desarrollar en estudiantes competencias no solo específicas, sino genéricas o transversales vinculadas con su desarrollo integral y con su inserción social. Este tema ha sido motivo de proyectos como Tuning Europa y, basado en éste, Tuning en América Latina. Lo que hoy en día está cuestionado es la eficacia de la Universidad y de la Educación para promover el desarrollo económico, la igualdad y la movilidad social, y en estos apartados se va a incidir a continuación. 


\subsection{Perspectivas críticas}

Los enormes esfuerzos que realizan los estados y naciones al asignar una fracción importante de sus recursos económicos y humanos a la educación, genera una expectativa que, algunas veces, es defraudada. Igualmente, la ciudadanía invierte una gran parte de su vida, ilusiones y recursos en su educación, obteniendo resultados que, en muchas ocasiones, no responden a las ilusiones y expectativas puestas en el proceso educativo.

Muchas son las causas de estas posibles decepciones, y es imposible abordarlas todas. No obstante, vamos a centrarnos en algunos aspectos. Comenzamos con la visión de economistas como Stiglitz y Greenwald (2015), que consideran que en la economía se estima necesaria la educación para incrementar el capital humano e incrementar el conocimiento de la humanidad y de las personas, y este incremento se suele medir por años de escolaridad. Ellos matizan, de una forma crítica, esta creencia, al afirmar que los años invertidos en aprendizajes memorísticos podrían no aumentar ni la productividad, ni lo que ellos denominan reserva de conocimiento. Este tipo de escolaridad no incrementa la capacidad de aprender permanentemente, y hasta podría impedirlo. Para estos autores, las deficiencias del sistema educativo son muy relevantes, puesto que consideran que el ritmo de aprendizaje (innovación), es el factor más determinante para los aumentos en la calidad de vida en las sociedades.

Desde el punto de vista de la pedagogía, Pérez Gómez (2012) aglutina una serie de críticas sobre las prácticas educativas, y ofrece alternativas para evitar el fracaso de la labor de las instituciones y sistemas educativos, y adaptarse a las nuevas demandas de un entorno cambiante y tecnológico. Este autor, señala también a la educación como clave, y señala que sobre ella reposan muchas de las esperanzas y expectativas, especialmente en los sistemas democráticos. Pero, igualmente, recoge una problemática respecto a la igualdad de oportunidades, y afirma, a partir de investigaciones e informes lo siguiente:

Que el factor que explica y predice en la actualidad de manera más significativa las diferencias en el rendimiento académico está configurado por las desigualdades socioculturales del contexto familiar ¿Dónde está pues la función compensatoria de la escuela si las diferencias socioculturales de origen explican en gran medida las diferencias de rendimiento de los estudiantes al acabar la etapa obligatoria? (Pérez Gómez, 2012: 79).

Sobre la desigualdad son también interesantes las apreciaciones del premio nobel de economía Tirole (2017), que considera que hay un riesgo de que aumente la desigualdad debida a la vinculación familiar de las personas y a la calidad de la educación.

Milanovic (2017) también recoge la posible reducción del valor de la educación, como explicación de los ingresos salariales, en una sociedad donde todas las personas estén bien educadas, y estima que las grandes desigualdades salariales dependerán más de la suerte, las fortunas familiares y los contactos sociales.

Desde la sociología rescatamos las críticas de Bauman (2013) que, aunque están insertas en un contexto de crisis económica en Europa, reflejan grandes inquietudes 
y desesperanzas. Bauman recoge las creencias generalizadas de la ciudadanía de que cada generación viviría mejor que la anterior, y que la juventud y las nuevas generaciones superarían los éxitos de sus antecesores (como sociedad). Asimismo, señala cómo la juventud y sus familias hicieron inversiones y solicitaron créditos para formación, porque creían que un título universitario era la llave para entrar en el sistema, y que en lo alto del escalafón social había sitio para todas las personas. Es decir, que los estudios garantizaban un buen empleo y éxito. Frente a todas esas ilusiones, la población egresada universitaria se encuentra, actualmente, con el desempleo, aceptando subempleos, salarios bajos, y con empleos parciales, temporales e inseguros, y muchos de ellos con créditos bancarios que no pueden devolver. Los efectos de la crisis económica, la situación de la población egresada y la falta de eficacia de la educación le lleva a hacer una demoledora crítica a la situación actual:

En nuestras sociedades dirigidas por la información y en las que se da por supuesto que el motor de las economías es el conocimiento -en una sociedad como ésta, cuyos éxitos económicos se consiguen gracias a la educación-, parece que el saber está fracasando, pues ya no garantiza el éxito, mientras que la educación también fracasa a la hora de cumplir su función: impartir ese saber. La visión de una movilidad social ascendente guiada por la educación, que neutralice las toxinas de la desigualdad haciéndolas soportables y convirtiéndolas en inofensivas, y la aún más desastrosa visión de la educación utilizada como medio para mantener en activo la movilidad social ascendente, son ahora dos visiones que están empezando a evaporarse de forma simultánea. Y con su desaparición, aquella excusa, propiciada y usada de común por nuestra sociedad en su esfuerzo por justificar sus injusticias se va a encontrar en un serio aprieto. (Bauman, 2013: 81-82).

La UNESCO (2013), también recoge que la relación causal entre educación y empleo, que ha justificado la inversión en "capital humano", está cuestionada por el alto nivel de desempleo de la población egresada de algunos países de Europa. Igualmente, reconoce la escasez de empleos cualificados que provoca una frustración en la juventud egresada y en sus familias, no solo en los países en desarrollo, sino también en países del Sur.

\subsection{La discusión en Latinoamérica}

¿Pero las situaciones señaladas se mantienen en América Latina? Para Senen et al. (2015), las universidades latinoamericanas son clave para el crecimiento económico de las zonas donde están ubicadas, debido a la formación de capital humano de alto nivel, la importancia de la investigación y desarrollo que realizan (ejecutan más de un tercio de la inversión en $\mathrm{I}+\mathrm{D})$, y su apoyo al emprendimiento y a la innovación.

Según un estudio, del PNUD (2014), de las Naciones Unidas, que recoge datos de ocho países de América (Argentina, Brasil, Chile, Colombia, Costa Rica, México, Perú y Uruguay), el ingreso laboral de la población egresada universitaria, comprendida entre 25 y 44 años, respecto a las personas del mismo intervalo de edad que han terminado los estudios secundarios, oscila entre 3 veces superior en 
Colombia y Chile y las 1,7 veces de Argentina y Uruguay. En México la diferencia es cercana al 2,5. Con estos datos parece que en América Latina la juventud universitaria tiene una importante diferencia de salarios, que retribuye su formación y esfuerzo.

Los datos de la OCDE (2016) apuntan la misma tendencia de recompensa por los estudios universitarios. En su informe afirma que el nivel educativo tiene una mayor relación con los ingresos en América Latina y el Caribe que en los países que pertenecen a la OCDE. Las importantes diferencias salariales, en función de la formación, son una fuente de desigualdad, y reflejan que aún hay carencias de trabajadores con alto nivel educativo. Igualmente, afirma que el $90 \%$ de la juventud con educación terciaria tiene empleos formales, frente al 30\% de quienes tienen estudios primarios.

América Latina sigue siendo la región con mayor índice de desigualdad del planeta (Universia-CINDA, 2016), y conforme al Coeficiente Gini (mide la desigualdad), 6 países iberoamericanos superan el coeficiente de 0,5 ; es decir, son en los que hay mayor desigualdad. Lo relevante es que en esta lista negativa están algunos de los países más desarrollados económicamente y con mejores universidades, como son Brasil y Chile, y muy cercano al 0,5 se encuentra México. Estos datos son coherentes con la posición de Hopenhayn (2016) que considera que la educación ha sido la mejor herramienta para la movilidad social, pero sigue reproduciendo la desigualdad. Ello presupone que la población egresada universitaria mejora su posición social, al recibir sueldos mucho más altos que la no universitaria, y esta gran diferencia es la que permite que la desigualdad sea muy amplia.

La creencia en la educación como respuesta a la desigualdad ha tenido un gran peso en la región, pero este avance hacia la igualdad no se ha producido porque las enormes diferencias "socioeconómicas de los estudiantes, sus familias y sus comunidades de origen condicionan severamente las oportunidades educativas de acceso, permanencia y aprovechamiento" (Saraví, 2015: 58).

No obstante, y aún reconociendo la desigualdad, se insiste en la necesidad de la educación. Henríquez (2014), Director del Instituto Internacional para la Educación Superior en América Latina y el Caribe vinculado a la UNESCO, en su presentación de un Informe, propone propiciar una inclusión, en los sistemas educativos, que abarque todos los niveles (incluida la Educación Superior), de los grupos sociales marginados y grupos étnicos. Esta inclusión debe conllevar que la juventud no abandone los estudios por razones extraeducativas y que no solo ingresen, sino que continúen y finalicen sus estudios. Por tanto, se confía en la educación como herramienta de inclusión y equidad. En este mismo informe de la UNESCO se afirma "que la Universidad latinoamericana reproduce fielmente las desigualdades de las sociedades, observándose casos extremos en cada uno de los países, especialmente en aquellos con poblaciones indígenas y afro-descendientes numerosas" (Albornoz, 2014: 23).

En el Informe Universia-CINDA (2016) se recogen y tratan 10 propuestas y claves estratégicas para las universidades latinoamericanas. Resulta relevante que entre ellas se señale la creación de instrumentos para la inserción laboral de los titulados universitarios, y para captar las demandas sociales. Igualmente, y también muy vinculada con nuestra investigación, está la propuesta de atender a las 
expectativas de la juventud universitaria, con objeto de que la formación que reciben en las titulaciones universitarias no defrauden las necesidades de inserción laboral y emprendimiento de la juventud universitaria.

En el caso de México, el porcentaje de población, entre 25 y 34 años con Educación Superior es del 25\%, considerado un nivel de masificación medio-bajo. No obstante, si se tiene en cuenta el nivel de desarrollo del país, y se compara con los otros países latinoamericanos (como Panamá, Perú, Ecuador o Paraguay) tiene un nivel bajo de masificación de estudiantes universitarios. Es decir, que en otros países con un menor nivel de desarrollo, hay una mayor masificación o porcentaje de estudiantes universitarios (Universia-CINDA, 2016).

No obstante, hay que destacar que a pesar del nivel de masificación medio-bajo, la evolución de matrícula universitaria ha tenido un fuerte incremento en México, que ha pasado de 1.962 .763 estudiantes en el 2000 a 2.867 .763 estudiantes en el 2010 lo que supone un incremento de 884.612 estudiantes en una década (Universia-CINDA, 2016). Asimismo, los datos aportados por la Asociación Nacional de Universidades e Instituciones de Educación Superior de México (ANUIES, 2017), ofrece una cifra de 3.762 .679 estudiantes, suponiendo un incremento de 915.303 estudiantes. Estos datos arrojan un fuerte incremento de estudiantes universitarios en México que casi ha duplicado su matrícula en 16 años.

$\mathrm{Si}$ atendemos a aspectos sociales, el porcentaje de hombres y mujeres, en México, se distribuían en un $50 \%$ en el año 2014. Por otro lado, el acceso a la Educación Superior tiene una alta dependencia del origen socioeconómico del joven. La brecha en América Latina y el Caribe, dándose una brecha media de un $35 \%$, entre estudiantes que provienen del quintil más rico con los del quintil más pobre. En el caso de de México, el $15.6 \%$ de la juventud universitaria proviene del quintil más pobre; por otro lado, frente al $46 \%$ que procede del quintil más rico (Universia-CINDA, 2016).

En México, volviendo de nuevo a la investigación de Saraví (2015), la calidad de los trabajos y el acceso rápido al empleo de las personas egresadas, además de por la formación, está condicionada por la clase social a la que pertenecen y sus redes familiares y de amistades. Es lo que el autor denomina capital sociocultural del alumnado. Quizás esta realidad pueda explicar por qué en México hay una menor masificación de estudiantes universitarios. Por otro, lado aunque los datos disponibles no son muy actuales, ya que datan del 2011, el porcentaje de personas desempleadas en México, que tienen educación superior es del 24,8\% (Banco Mundial, 2016), probablemente este porcentaje proceda de la población egresada que pertenece de los quintiles más pobres.

Por último, frente al pesimismo de los europeos, respecto al futuro, Trejo (2016) afirma que la juventud en Iberoamérica, identifica con claridad los conflictos de la sociedad, pero son más optimistas que las personas adultas y tienen "una mayor capacidad de resiliencia que les permite adaptarse a las transformaciones de una región que seguirá en continuo y acelerado cambio" (Trejo, 2016: 151).

Estas investigaciones y debates abiertos nos llevan a comprobar en qué medida coinciden estas posiciones con las vivencias de la población egresada.

Como objetivo general de este trabajo se pretende indagar si la juventud universitaria mexicana cuenta con el perfil profesional que exigen los retos y las expectativas de la realidad social actual. En este sentido, se tratará de analizar si la 
formación universitaria contribuye al empoderamiento laboral y social de la juventud mexicana. Como objetivo específico se pretende ponderar la relevancia relativa de algunos de los factores que influyen en el desarrollo de competencias durante las trayectorias formativas. De manera complementaria, se investigará si la formación universitaria recibida faculta a la población egresada para el empoderamiento social, laboral y económico. Para ello será necesario construir, validar y aplicar un instrumento (objetivos operativos), que sea capaz de evaluar las competencias trabajadas durante el periodo formativo de la juventud universitaria. Como objetivo añadido se pretende que los resultados del estudio permitan orientar las líneas estratégicas de la Universidad de cara a fortalecer los currícula académicos de los centros universitarios objeto de estudio.

\section{Metodología}

Este trabajo es parte de una investigación más amplia, que emplea el método mixto. En este artículo se presenta la fase de la investigación en la que se diseñó, validó y aplicó un cuestionario a una muestra piloto de 218 personas egresadas. En este sentido se puede decir que en este trabajo se emplea la metodología de encuestas.

Para el diseño del cuestionario se tuvo en cuenta una amplia revisión de la literatura (p. ej. Ernest, Matthew y Samuel, 2015; OCDE/CEPAL/CAF, 2016; Roman y Maxim, 2017). El cuestionario diseñado fue validado por 10 jueces, expertos en metodología de investigación y en ámbito de emprendimiento, que valoraron la idoneidad del contenido de cada uno de los ítems (validez de contenido). Algunas sugerencias fueron admitidas y el instrumento definitivo se configuró con 70 ítems que combinaban varios formatos: preguntas de identificación, de elección múltiple y una escala Likert (de 1 a 5).

A partir de los 14 ítems de la escala Likert se realizó un estudio de consistencia interna (fiabilidad) obteniéndose un Alpha de Cronbach de 0,90. Sobre estos mismos ítems se realizó un análisis factorial exploratorio, con el método de componentes principales. El análisis factorial se consideró factible a partir del cumplimiento de las condiciones de aplicación $(\mathrm{KMO}=0,89$, y Prueba de esfericidad de Barlett con un $\chi^{2}$ de 2063,61 g. 1. y $p<0.0005$. El análisis consiguió una solución factorial de 2 componentes (factores) que explicaban el $69,77 \%$ de la variabilidad. Uno de los factores reunía ítems relacionados con las competencias adquiridas y la formación para el desempeño fuera de la Universidad, y el segundo factor congregaba las variables relacionadas con la coherencia entre la formación obtenida en la Universidad y las actividades desarrolladas en la actualidad. Similares indicadores han sido empleados en estudios con cuestionarios como el de Ernest, Matthew y Samuel (2015).

La muestra piloto estuvo formada por 218 personas egresadas $(38,5 \%$ hombres y $61,5 \%$ mujeres) con una edad media de 26.73 años $(\mathrm{DS}=3,02)$. El muestreo se ha realizado de manera incidental. Se empleó este tipo de muestreo no probabilístico para incluir en la muestra a la juventud egresada que cumpliera con los criterios de selección establecidos (carreras universitarias similares en los diversos centros universitarios y mismo periodo de egreso 2013-2017), y los 
objetivos de la investigación. A nivel institucional, los centros universitarios se dirigieron a su población egresada respetando la ley de protección de datos de México, pidiéndole que rellenara el cuestionario online de manera anónima. El perfil de la persona egresada se encuentra dividido en dos mitades, con personas que se han independizado (viven ya solas, o con sus parejas, e incluso tienen hijos) en un $50 \%$, o que, en ese mismo porcentaje, viven aún con sus familias. Los participantes en la encuesta finalizaron todos su carrera entre 2013 y 2017, con una media de finalización de 2014,67 (DS =1,43), y en su mayoría ya cuentan con un empleo $(88,5 \%)$. En la tabla 1 se observa la distribución por títulos universitarios que tiene la muestra egresada.

Tabla. 1. Títulos universitarios de la muestra egresada.

\begin{tabular}{|l|l|l|}
\hline & Frecuencia & Porcentaje \\
\hline Agronegocios & 5 & 2,3 \\
\hline Comunicación Pública & 31 & 14,2 \\
\hline Desarrollo Turístico Sustentable & 7 & 3,2 \\
\hline Letras Hispánicas & 10 & 4,6 \\
\hline Periodismo & 59 & 27,1 \\
\hline Sistemas de Información & 42 & 19,3 \\
\hline Trabajo Social & 10 & 4,6 \\
\hline Turismo & 54 & 24,8 \\
\hline Total & 218 & 100 \\
\hline
\end{tabular}

Fuente: Elaboración propia

Se realizaron análisis descriptivos de la muestra (frecuencias y porcentajes, medias y desviaciones típicas) para analizar la importancia relativa de las diferentes variables consideradas. También se realizaron análisis de varianza (ANOVA), y análisis multivariante de la varianza (MANOVA), para buscar diferencias de opiniones en la muestra egresada entre los ítems del cuestionario y las variables género, tipo de empresa en la que trabajan (pública o privada), y tamaño de la empresa. Análisis similares han sido realizados en otros estudios sobre esta misma temática como el de Roman y Maxim (2017). Para comprobar la igualdad de las matrices de covarianzas se usó la prueba de Box. También se empleó el contraste de Levene sobre igualdad de varianzas error para contrastar las hipótesis de que la varianza de error de las variables dependientes era igual en los diversos grupos. Tras los contrastes univariantes y multivariantes se realizaron pruebas post hoc HDS de Tukey (comparaciones múltiples) para determinar en qué grupos concretos se observaban diferencias. Los análisis se realizaron con el paquete estadístico SPSS v22.0. 


\section{Resultados}

Algunos de los rasgos esenciales que caracterizan la muestra son los siguientes. La mayoría trabaja en empresas privadas $(63,3 \%)$, y desarrolla alguna función directiva o de mando (48,6\%). De los que trabajan en empresas privadas, el $11,9 \%$ lo hacen en una empresa propia (emprendimiento).

El 80,8\% está trabajando en la misma región/estado (Jalisco) en el que estudió su carrera. Solo el $5 \%$ desarrolla sus actividades laborales en otros estados (fuera de Jalisco), y el $3.2 \%$ en otros países.

En la tabla 2 se muestra el tamaño de empresa en la que se trabaja. Como se observa en la misma, los mayores porcentajes $(36,2 \%)$ trabajan en grandes empresas y en medianas (18.3\%). Las microempresas (plantilla entre 2 y 10 ) suponen la tercera opción con un $13.8 \%$. Las personas trabajadoras por cuenta propia, sin personal, suponen un $6 \%$.

Tabla. 2. Tipo de empresa en función del tamaño.

\begin{tabular}{|l|l|l|}
\hline & Frecuencia & Porcentaje \\
\hline Gran empresa (más de 250) & 79 & 36,2 \\
\hline Mediana empresa (entre 50 y 250) & 40 & 18,3 \\
\hline Pequeña empresa (entre 11 y 49) & 20 & 9,2 \\
\hline Microempresa (plantilla entre 2 y 10) & 30 & 13,8 \\
\hline Independiente (sin personal) & 13 & 6 \\
\hline NS/NC & 20 & 9,2 \\
\hline Total & 218 & 100 \\
\hline
\end{tabular}

Fuente: Elaboración propia

En la tabla 3 se recogen los resultados, de los ítems del cuestionario, que se presentaron en una escala tipo Likert ( 0 es nada de acuerdo y 5 totalmente de acuerdo). En general, destaca que todos los valores están por encima del valor medio de la escala $(2,5)$. Las desviaciones típicas son todas muy similares y van en un rango desde 1,33 a 1,77 . Los ítems con un acuerdo mayor (menor desviación típica de 1,33), son los relativos a que "las competencias adquiridas en la Universidad contribuyeron a tener una buena relación con los clientes o usuarios" (con una media alta de 3,72), y a que "la Universidad cumplió las expectativas sobre la formación como profesional" (con una media de 3,39). En ambos ítems, por el valor de la desviación típica, se puede indicar que sus valores medios son los más representativos del conjunto. El ítem en el que hay una mayor dispersión de opiniones $(1,77)$, es el referido a si la categoría o nivel de puesto en el que trabajo es acorde con la carrera realizada (media de 3,14 ).

El ítem con una valoración más alta es el relativo a la posibilidad de cambio de trabajo en los próximos 5 años (media de 3,75). También destacan en valores altos los relativos a que las competencias adquiridas en la Universidad contribuyeron a tener una buena relación con los clientes y usuarios (media de 3,72), con los compañeros de trabajo (3,57), con las amistades (media de 3,51), y con los 
familiares $(3,41)$. Otros ítems con puntuaciones altas son los relativos a que los resultados del trabajo son reconocidos por los superiores $(3,61)$, y la satisfacción con el trabajo actual $(3,47)$.

Tres de los ítems que valoran la Universidad (expectativas cumplidas, formación recibida para el empleo y competencias adquiridas), reciben altas valoraciones (medias de 3,39, 3,42 y 3,44 respectivamente). La muestra egresada indica también que la Universidad les prepara para afrontar los retos que impone la sociedad contemporánea (media de 3,00).

El ítem menos valorado (si bien está por encima del valor medio de la escala), es el relativo a la concordancia entre el salario recibido y el nivel de estudios $(2,74)$.

Tabla. 3. Valoración de los ítems del cuestionario (de mayor a menor).

\begin{tabular}{|l|l|l|}
\hline Ítem & Media & DS \\
\hline Seguramente cambiaré de trabajo en los próximos 5 años & 3,75 & 1,73 \\
\hline $\begin{array}{l}\text { Las competencias adquiridas en la Universidad contribuyeron a } \\
\text { tener una buena relación con los clientes o usuarios }\end{array}$ & 3,72 & 1,33 \\
\hline $\begin{array}{l}\text { Los resultados de mi trabajo son reconocidos por mis superiores } \\
\text { (en caso de tenerlos) }\end{array}$ & 3,61 & 1,63 \\
\hline $\begin{array}{l}\text { Las competencias adquiridas en la Universidad contribuyeron a } \\
\text { tener una buena relación con los companeros de trabajo }\end{array}$ & 3,57 & 1,37 \\
\hline $\begin{array}{l}\text { Las competencias adquiridas en la Universidad contribuyeron a } \\
\text { tener una buena relación con mis amistades }\end{array}$ & 3,51 & 1,42 \\
\hline $\begin{array}{l}\text { Estoy satisfecho con el trabajo que desempeño actualmente } \\
\text { En la Universidad adquirí las competencias, habilidades y } \\
\text { destrezas para desempeñar bien mi trabajo] }\end{array}$ & 3,47 & 1,56 \\
\hline $\begin{array}{l}\text { La formación que recibí en la Universidad fue importante para la } \\
\text { obtención del empleo }\end{array}$ & 3,42 & 1,39 \\
\hline $\begin{array}{l}\text { Las competencias adquiridas en la Universidad contribuyeron a } \\
\text { tener una buena relación con mis familiares }\end{array}$ & 3,41 & 1,44 \\
\hline $\begin{array}{l}\text { La Universidad cumplió mis expectativas sobre mi formación } \\
\text { como profesional }\end{array}$ & 3,39 & 1,33 \\
\hline $\begin{array}{l}\text { Existe correspondencia entre lo aprendido en la Universidad y la } \\
\text { actividad que realizo en mi lugar de trabajo }\end{array}$ & 3,22 & 1,55 \\
\hline $\begin{array}{l}\text { La categoría o nivel de puesto en el que trabajo es acorde a mi } \\
\text { carreara realizada en la Universidad }\end{array}$ & 3,14 & 1,77 \\
\hline $\begin{array}{l}\text { Considero que la Universidad prepara a su alumnado para } \\
\text { afrontar los retos que impone la sociedad contemporánea }\end{array}$ & 3,00 & 1,41 \\
\hline $\begin{array}{l}\text { El sueldo que percibo por mi trabajo es acorde a mi nivel de } \\
\text { estudios }\end{array}$ & 2,74 & 1,66 \\
\hline
\end{tabular}

Fuente: Elaboración propia 
En los análisis de varianza realizados no se encontraron diferencias significativas entre los ítems del cuestionario y las variables género y tipo de empresa (pública o privada). Si se detectaron varias diferencias entre algunos ítems del cuestionario y el tamaño de la empresa. Como se observa en la tabla 4, en las empresas medianas (plantilla entre 50 y 250), los resultados del trabajo son más reconocidos por los superiores que en las grandes empresas $(p=0,045)$. También lo son en relación a los trabajadores independientes (sin personal), aunque este resultado puede parecer obvio.

Otro resultado de la tabla 4 muestra como las personas trabajadoras de una microempresa (entre 2 y 10) valoran más la correspondencia entre lo aprendido en la Universidad y la actividad que realizan en el trabajo que los que trabajan de manera independiente. Finalmente, otro resultado significativo señala que en las pequeñas empresas (entre 11 y 49 trabajadores), piensan que la Universidad cumplió mejor las expectativas sobre la formación como profesionales que en las empresas medianas $(p=0,019)$.

Tabla. 4. Diferencias significativas entre ítems del cuestionario y la variable tamaño de la empresa.

\begin{tabular}{|c|c|c|c|c|c|}
\hline $\begin{array}{l}\text { Variable } \\
\text { Dependiente }\end{array}$ & $\begin{array}{l}\text { Tamaño de la } \\
\text { empresa (i) }\end{array}$ & $\begin{array}{l}\text { Tamaño de la } \\
\text { empresa (j) }\end{array}$ & $\begin{array}{l}\text { Diferencia } \\
\text { de medias } \\
(\mathrm{i}-\mathrm{j})\end{array}$ & $\begin{array}{l}\text { Error } \\
\text { estándar }\end{array}$ & $p$ \\
\hline \multirow{2}{*}{$\begin{array}{l}\text { Los resultados } \\
\text { de mi trabajo son } \\
\text { reconocidos por } \\
\text { mis superiores } \\
\text { (en caso de } \\
\text { tenerlos }\end{array}$} & $\begin{array}{l}\text { Mediana } \\
\text { empresa } \\
\text { (entre } 50 \quad \text { y } \\
250)\end{array}$ & $\begin{array}{l}\text { Independiente } \\
\text { (sin personal) }\end{array}$ & 1,13 & 0,55 & 0,041 \\
\hline & $\begin{array}{l}\text { Mediana } \\
\text { empresa } \\
\text { (entre } 50 \quad \text { y } \\
250 \text { ) }\end{array}$ & $\begin{array}{l}\text { Gran empresa } \\
\text { (más de 250) }\end{array}$ & 0,69 & 0,34 & 0,045 \\
\hline $\begin{array}{l}\text { Existe } \\
\text { correspondencia } \\
\text { entre lo } \\
\text { aprendido en la } \\
\text { Universidad y la } \\
\text { actividad que } \\
\text { realizo en mi } \\
\text { lugar de trabajo }\end{array}$ & $\begin{array}{l}\text { Microempresa } \\
\text { (plantilla } \\
\text { entre } 2 \text { y 10) }\end{array}$ & $\begin{array}{l}\text { Independiente } \\
\text { (sin personal) }\end{array}$ & 1,38 & 0,63 & 0,030 \\
\hline $\begin{array}{l}\text { La Universidad } \\
\text { cumplió mis } \\
\text { expectativas } \\
\text { sobre mi } \\
\text { formación como } \\
\text { profesional }\end{array}$ & $\begin{array}{l}\text { Pequeña } \\
\text { empresa } \\
\text { (entre } 11 \text { y } \\
49 \text { ) }\end{array}$ & $\begin{array}{l}\text { Mediana } \\
\text { empresa } \\
\text { (entre } 50 \quad \text { y } \\
250)\end{array}$ & 1,29 & 0,54 & 0,019 \\
\hline
\end{tabular}

Fuente: Elaboración propia 
Para realizar los contrastes multivariantes (MANOVA) se calcularon la traza de Pillai, la Lambda de Wilks, la traza de Hotelling y la raíz mayor de Roy. Las pruebas $F$ contrastan el efecto multivariante del tamaño de la empresa, y todas ellas se basan en comparaciones por pares linealmente independientes entre las medias marginales estimadas. En la tabla 5 se observa cómo todos los contrastes realizados están cerca de la significación, pero solo la raíz mayor de Roy se muestra como significativo $(p=0,001)$ y con el relativamente mayor tamaño del efecto $(0,20)$.

Tabla. 5. Pruebas de contraste de MANOVA para Tamaño de la Empresa.

\begin{tabular}{|l|l|l|l|l|l|l|l|}
\hline Efecto & $\begin{array}{l}\text { Contrast } \\
\text { e }\end{array}$ & Valor & $\mathrm{F}$ & $\begin{array}{l}\text { gl. de la } \\
\text { hipótesis }\end{array}$ & $\begin{array}{l}\text { gl. del } \\
\text { error }\end{array}$ & $p$ & $\begin{array}{l}\text { Tamaño } \\
\text { del } \\
\text { efecto }\end{array}$ \\
\hline $\begin{array}{l}\text { Tamaño } \\
\text { de la } \\
\text { empresa }\end{array}$ & $\begin{array}{l}\text { Traza de } \\
\text { Pillai }\end{array}$ & 0,43 & 1,32 & 56 & 608 & 0,067 & 0,11 \\
\hline & $\begin{array}{l}\text { Lambda } \\
\text { de Wilks }\end{array}$ & 0,63 & 1,33 & 56 & 581,75 & 0,062 & 0,11 \\
\hline & $\begin{array}{l}\text { Traza de } \\
\text { Hotelling }\end{array}$ & 0,51 & 1,34 & 56 & 590 & 0,057 & 0,11 \\
\hline & $\begin{array}{l}\text { Raíz } \\
\text { mayor de } \\
\text { Roy }\end{array}$ & 0,25 & 2,71 & 14 & 152 & 0,001 & 0,20 \\
\hline
\end{tabular}

Fuente: Elaboración propia

Para determinar en qué ítems concretos se producían las diferencias significativas entre los diversos tipos de empresas, según tamaño, se realizaron comparaciones múltiples (pruebas post hoc) a través de la DHS de Tukey. En la tabla 6 se incluyen solo los contrastes significativos entre los ítems del cuestionario y el tamaño de la empresa. Como se observa en dicha tabla, no existen muchas diferencias post hoc significativas. Tan solo dos de ellas entre microempresa y trabajadores independientes, y una entre mediana y gran empresa. Las personas que trabajan en microempresas piensan que existe mayor correspondencia entre lo aprendido en la Universidad y la actividad que realizan en el trabajo $(p=0,011)$. También se produce esa diferencia entre estos mismos tipos de empresas (a favor de las microempresas), y las opiniones de que las competencias adquiridas en la Universidad contribuyeron a tener una buena relación con los compañeros de trabajo $(p=0,014)$.

La última diferencia significativa se produce entre las opiniones de los trabajadores de las grandes empresas y los de las empresas medianas. Para los que trabajan en las grandes empresas, la Universidad cumplió mejor las expectativas sobre la formación como profesionales que para los que trabajan en empresas medianas $(p=0,034)$. 
Tabla. 6. Contrastes significativos en pruebas post hoc de Tukey. MANOVA entre ítems del test y tamaño de la empresa.

\begin{tabular}{|c|c|c|c|c|c|}
\hline $\begin{array}{l}\text { Variable } \\
\text { Dependiente }\end{array}$ & $\begin{array}{l}\text { Tamaño de la } \\
\text { empresa (i) }\end{array}$ & $\begin{array}{l}\text { Tamaño de la } \\
\text { empresa (j) }\end{array}$ & $\begin{array}{l}\text { Diferencia } \\
\text { de medias } \\
(\mathrm{i}-\mathrm{j})\end{array}$ & $\begin{array}{l}\text { Error } \\
\text { estándar }\end{array}$ & $p$ \\
\hline $\begin{array}{l}\text { Existe } \\
\text { correspondencia } \\
\text { entre lo } \\
\text { aprendido en la } \\
\text { Universidad y } \\
\text { la actividad que } \\
\text { realizo en mi } \\
\text { lugar de trabajo }\end{array}$ & $\begin{array}{l}\text { Microempresa } \\
\text { (plantilla } \\
\text { entre } 2 \text { y } 10 \text { ) }\end{array}$ & $\begin{array}{l}\text { Independiente } \\
\text { (sin personal) }\end{array}$ & 1,60 & 0,49 & 0,011 \\
\hline $\begin{array}{l}\text { Las } \\
\text { competencias } \\
\text { adquiridas en la } \\
\text { universidad } \\
\text { contribuyeron a } \\
\text { tener una buena } \\
\text { relación con los } \\
\text { compañeros de } \\
\text { trabajo }\end{array}$ & $\begin{array}{l}\text { Microempresa } \\
\text { (plantilla } \\
\text { entre } 2 \text { y } 10 \text { ) }\end{array}$ & $\begin{array}{l}\text { Independiente } \\
\text { (sin personal) }\end{array}$ & 1,25 & 0,45 & 0,014 \\
\hline $\begin{array}{l}\text { La Universidad } \\
\text { cumplió mis } \\
\text { expectativas } \\
\text { sobre } \\
\text { formación } \\
\text { como } \\
\text { profesional }\end{array}$ & $\begin{array}{l}\text { Gran empresa } \\
\text { (más de 250) }\end{array}$ & $\begin{array}{l}\text { Mediana } \\
\text { empresa } \\
\text { (entre } 50 \quad \text { y } \\
250)\end{array}$ & 0,721 & 0,25 & 0,034 \\
\hline
\end{tabular}

Fuente: Elaboración propia

\section{Conclusiones}

Con los datos e información aportados se puede destacar que más de un tercio de la juventud egresada de la Universidad obtiene empleo en las grandes empresas, que sumado a los que trabajan en medianas empresas llegan al 54,5\% de la muestra. En general, en las grandes y medianas empresas los salarios son más altos y las posibilidades de ascender a puestos de mayor responsabilidad, con contratos más estables y nivel salarial son grandes. Teniendo en cuenta que en México hay un alto nivel de actividad económica no formal, y que la mayoría de los empleos legalizados están en las pequeñas y microempresas, los datos de la muestra manifiestan una integración de calidad en el mercado laboral. 
Por un lado, respecto a las competencias adquiridas, la juventud egresada, destaca las relacionadas con habilidades sociales, frente a la formación para las actividades relacionadas con sus empleos. Esto nos sitúa en un debate sobre si las competencias que se deben desarrollar más son las específicas o las genéricas. Para Stiglitz y Greenwald (2016), las capacidades de aprendizaje pueden ser específicas o generales, ellos apuestan por mejorar las específicas si estamos en un "nicho específico" donde haya convergencia de empleos del mismo tipo. Las competencias genéricas son más útiles en una sociedad de alto nivel.

Por otro lado, la crítica a los salarios y a la categoría laboral en relación a su formación, sintoniza con la que evidencia una tendencia hacia la pérdida económica de la clase media y de los salarios de la juventud egresada universitaria. Esta evidencia ha sido reflejada en las críticas expresadas anteriormente (Bauman, 2013; Milanovic, 2017), y de otros autores (Bauman y Bordini, 2016, Castells, 2012; Touraine, 2013), relacionada con la crisis en los paises desarrollados y sus efectos en la juventud. Quizás estas críticas de la juventud egresada sea un indicio de la propagación rápida de esas problemáticas, incluso a lugares donde aún no hay un elevado número de personas con estudios universitarios, y por tanto, no hay una sobresaturación de personas trabajadoras con alto nivel de formación.

Además, el alto porcentaje de personas egresadas que piensan que cambiarán de puesto laboral, dado que han terminado sus estudios recientemente (cinco años o menos), puede responder a un reajuste o acomodación para acercarse más a un puesto con mayor relación con su formación, o bien, puede ser como señala Bauman (2004), una de las señales de lo que él denomina "modernidad líquida", caracterizada porque nada es estable, ni dura mucho en el tiempo.

No queremos terminar sin ratificar que las respuestas de la juventud egresada nos señalan que los que trabajan en microempresas estiman que su trabajo está más vinculado con su formación. Esto puede deberse a que, las microempresas, apuesten más por trabajos más especializados y busquen a personas egresadas vinculadas a esa especialización, frente a las grandes y medianas empresas. Resultados como éste, necesitan de más datos y más investigación para ser contrastados.

Concluimos pues, señalando que juventud egresada de la Universidad de Guadalajara considera que la formación universitaria recibida le faculta para el empoderamiento social y, en menor medida, para el laboral y económico.

Los datos y respuestas igualmente, señalan que hay diferencias importantes entre la percepción de la juventud universitaria egresada de los países desarrollados, especialmente de los europeos, pero que parece que hay indicios, a pesar del mayor optimismo de la juventud iberoamericana, que en un corto plazo puede acercarse a la visión y realidad pesimista. Esto sería un grave riesgo para México y para la región, porque teniendo aún una importante carencia de trabajadores, altamente cualificados, y de profesionales vinculados a la investigación e innovación y dentro de la era del conocimiento, una falta de motivación de la juventud, para acceder a los estudios de Educación Superior, podría frenar el crecimiento y desarrollo en el sentido amplio de México, y comprometer gravemente la convergencia con los países con un mayor nivel de desarrollo humano y bienestar. 
Aunque los resultados obtenidos señalan que la formación universitaria de la muestra egresada ha mejorado su empoderamiento social, laboral y económico (estos dos últimos en menor medida), resulta necesario aumentar el tamaño de la misma para que las conclusiones posean mayor relevancia. Así, un incremento representativo de la muestra, y la diversificación del número de títulos universitarios considerados, en estos y en otros centros universitarios, puede ilustrar un escenario de egreso más amplio, que a su vez enriquezca las conclusiones de la investigación. Otras líneas de investigación que pueden aportar información de relevancia, y profundizar en el conocimiento del empoderamiento laboral y social de la juventud universitaria mexicana, son el uso de metodologías cualitativas para analizar el estudio de informes institucionales realizados sobre este tema en diversas regiones y universidades del país, y la inclusión en el diseño de investigación de entrevistas a egresados y a cargos de responsabilidad en los centros universitarios relacionados con la empleabilidad de las poblaciones universitarias. En estas cuestiones, el equipo de investigación ya está trabajando, con el objetivo de poder ofrecer resultados y conclusiones de aún mayor calado a medio plazo, que ayuden a las propias instituciones universitarias a orientar sus políticas formativas en relación al empleo de sus poblaciones egresadas.

\section{Referencias bibliográficas}

Albornoz, O. (2014) La educación superior pública y privada en AL\&C: las presiones del Estado, el mercado y la sociedad, una perspectiva contemporánea. En VVAA, La Educación Superior Pública y Privada en América Latina y el Caribe. Contexto de Internacionalización y proyecciones de políticas públicas (pp. 16-60). UNESCO e Instituto Internacional para la Educación Superior en América Latina y el Caribe (IESALC). Disponible en http://www.iesalc.unesco.org.ve/index.php?option=com fabrik\&view=details\&formid= 2\&rowid $=173$ \&lang $=$ es.

ANUIES (2017) Anuarios estadísticos de Educación Superior. Ciclo escolar 2016-2017. Disponible en http:/www.anuies.mx/informacion-y-servicios/informacion-estadisticade-educacion-superior/anuario-estadistico-de-educacion-superior.

Banco Mundial (2016) World Development Indicators 2016. Disponible en http://documentos.bancomundial.org/curated/es/805371467990952829/Worlddevelopment-indicators-2016.

Barro, S. et al. (2015) La transferencia de I+D, la innovación y emprendimiento en las universidades. Educación Superior en Iberoamérica. Informe 2015. Santiago de Chile: Redemprendia, Universia y CINDA. Disponible en https://www.redemprendia.org/sites/default/files/descargas/informeTransferenciaI\%2B D2015.pdf.

Bauman, Z. (2004) Modernidad líquida. México. Fondo de Cultura Económica.

Bauman, Z. (2013) Sobre la educación en un mundo líquido. Barcelona: Paidós.

Bauman, Z. y Bordini, C. (2016) Estado de crisis. México: Paidós.

Castells, M. (2012) Redes de indignación y esperanza. Los movimientos sociales en la era de Internet. Madrid: Alianza Editorial. 
Díaz Domínguez, T. y Alemán, P. A. (2008) La educación como factor de desarrollo. Revista Virtual Universidad Católica del Norte, $\mathrm{N}^{\mathrm{o}}$ 23, pp. 1-15. Disponible en http://www.redalyc.org/pdf/1942/194220391006.pdf.

Ernest, K., Matthew, S. K. y Samuel, A. K. (2015) Towards entrepreneurial learning competencies: The perspective of built environment students. Higher Education Studies, $\mathrm{N}^{\mathrm{0}}$ 5, Vol. 1, pp. 20-30.

Gimeno, J. (2013) En busca del sentido de la educación. Madrid: Ediciones Morata.

Henríquez, P. (2014) Presentación. En VVAA, La Educación Superior Pública y Privada en América Latina y el Caribe. Contexto de Internacionalización y proyecciones de políticas públicas (pp. 3-15). UNESCO e Instituto Internacional para la Educación Superior en América Latina y el Caribe (IESALC). Disponible en http://www.iesalc.unesco.org.ve/index.php?option=com_fabrik\&view=details\&formid= 2\&rowid=173\&lang=es.

Hopenhaym, M. (2016) Desafíos de la educación para una agenda emancipatoria: reflexiones desde el entorno iberoamericano. Revista Pensamiento Iberoamericano. Juventud, emprendimiento y Educación, $\mathrm{N}^{\mathrm{o}} 3$, Vol. 2, pp. 45-52. Disponible en https://www.segib.org/wp-content/uploads/06-MH.pdf.

Larrañaga, O. y Rodríguez, M. E. (2014) Clases medias y educación en América Latina. Programa de las Naciones Unidas para el Desarrollo. Disponible en http://www.cl.undp.org/content/dam/chile/docs/pobreza/undp_cl_pobreza_clases_media s_2014.pdf.

Milanovic, B. (2017) Desigualdad mundial. Un nuevo enfoque para la era de la globalización. México: Fondo de Cultura Económica.

OCDE/CEPAL/CAF (2016) Perspectivas Económicas de América Latina 2017. Juventud, competencias $y$ emprendimiento. Paris: OCDE Publishing. Disponible en https://www.oecd.org/dev/americas/E-book_LEO2017_SP.pdf.

Pérez Gómez, A. I. (2012) Educarse en la era digital. Madrid: Ediciones Morata.

Roman, T. y Maxim, A. (2017) National culture and higher education as pre-determining factors of students entrepreneurship. Studies in Higher Education, No 42, Vol. 6, pp. 993-1014.

Saraví, G.A. (2015) Juventudes fragmentadas. Socialización, clase y cultura de la construcción de la desigualdad. México: FLACSO México y CIESAS.

Soto, J. L. y Torres, C. A. (2016) Percepciones y expectativas del aprendizaje en jóvenes universitarios. Revista de Docencia Universitaria, No 14, Vol. 1, pp. 51-57.

Stiglitz, J. E. y Greenwald, B. C. (2015) La creación de una sociedad del aprendizaje. México: Crítica M. R.

Tirole, J. (2017) La economía del bien común. Barcelona: Taurus.

Taatila, V. P. (2010) Learning Empreneurship in Higher Education. Education \& Training, $\mathrm{N}^{\mathrm{o}}$ 52, Vol. 1, pp. 48-61.

Touraine, A. (2013) Después de la crisis. México: Fondo de Cultura Económica.

Trejo, M. (2016) Expectativas de las personas jóvenes en Iberoamérica. Revista Pensamiento Iberoamericano. Juventud, emprendimiento y Educación, № 3, Vol. 2, pp. 144-152. Disponible en https://www.segib.org/wp-content/uploads/016-MTC.pdf.

UNESCO (2009) Conferencia Mundial sobre la Educación Superior: La nueva dinámica de la Educación Superior y la Investigación para el cambio social y el desarrollo. Disponible en http://www.unesco.org/education/WCHE2009/comunicado_es.pdf.

UNESCO (2013) Replantear la educación en un mundo en mutación. Disponible en https://es.scribd.com/document/216633008/Educacion-Para-Un-Mundo-en-Mutacion. 
Universia y Centro Universitario de Desarrollo-CINDA (2016) Educación Superior en Iberoamérica. Informe 2016. Santiago de Chile: CINDA. Disponible en https://www.cinda.cl/download/libros/CINDA-2012-Informe-de-Educación-SuperiorINTERIOR-101\%20(1).pdf.

Universidad de Guadalajara (2018) Numeralia. Disponible en http://www.copladi.udg.mx/estadistica/numeralia. 